\title{
Die Zukunft der Chirurgischen Onkologie ${ }^{1}$
}

\author{
S. Post \\ Universitätsklinikum Mannheim
}

\section{Allgemeines}

Die Fragestellung des Titels läßt sich in zwei grundsätzlich verschiedenen Dimensionen explorieren: zum einen als Frage nach der Zukunft des Chirurgen innerhalb der Onkologie, zum anderen als Frage nach dem künftigen Stellenwert der Operation innerhalb der Onkologie. Auch wenn der Schwerpunkt der folgenden Ausführungen auf letzterem liegen soll, seien einige grundsätzliche Überlegungen zur erstgenannten Fragestellung erlaubt, in die vielfältige - nicht zuletzt berufspolitische - Überlegungen einfließen.

Die moderne Onkologie ist ihrem Wesen nach eine zunehmend interdisziplinäre Aufgabe, die von den drei großen Disziplinen Chirurgie, Innere Medizin und Strahlentherapie dominiert wird. Jede Disziplin erhebt Ansprüche auf unterschiedliche Teilaspekte der multimodalen Therapie, wobei Überschneidungen und Konkurrenz nicht ausbleiben. Dennoch kann kaum bestritten werden, daß die operative Therapie die Domäne des chirurgischen Onkologen, die Chemotherapie die Domäne des internistischen Onkologen und die Strahlentherapie die Domäne des Radio-Onkologen ist. Für den einzelnen Patienten ist es sicherlich wenig ersprießlich, wenn sich verschiedene Disziplinen um Teilaspekte der multimodalen Therapie streiten. Andererseits kann vielerorts ein multimodales Therapiekonzept für den Patienten überzeugender vertreten werden, wenn zumindest die koordinierende Funktion in der Hand eines Haupt-Therapeuten liegt. Bei (neo-)adjuvanten Therapien fällt diese Rolle häufig dem Operateur zu.

Dessen ungeachtet besteht innerhalb der einzelnen Hauptdisziplinen die Tendenz zu weiterer Subspezialisierung (z.B. hepato-biliäre Chirurgie, kolorektale Chirurgie ...), nicht zuletzt deshalb, weil mit zunehmender Verfeinerung der Techniken auch die Ausbildungsansprüche steigen. Je enger das organoder tumorbezogene Spektrum des einzelnen Therapeuten ist, um so eher ist der Anspruch auf Konzentration mehrerer Modalitäten in einer Hand glaubhaft. So haben traditionell operative Disziplinen mit engerem Spektrum (z.B. Gynäkolo-

\footnotetext{
${ }^{1}$ Nach einer «Special lecture», gehalten am 6. Februar 1999 anläßlich der 15. Jahrestagung der «Association for Oncology of the Upper Rhine».
}

gie oder Urologie) ein festes Standbein in der Chemotherapie. Wenig überzeugend für Kollegen wie Patienten und nicht mehr zeitgemäß erscheinen Ansprüche einzelner Therapeuten auf zugleich breite (viele verschiedene Organ-/Tumorentitäten umfassende) wie tiefe (viele Therapie-Modi umfassende) onkologische Kompetenz. Entscheidend für die Zuständigkeit muß aber vielerorts die individuell belegte Befähigung des einzelnen Onkologen sein. Der unaufhaltsame Trend zur internationalen Harmonisierung wird in naher Zukunft auch bei uns eine stärkere Subspezialisierung fördern und manche Erbhöfe in Frage stellen.

Mit diesen allgemeinen Bemerkungen und einem ausdrücklichen Bekenntnis zur interdisziplinären Kooperation im Rahmen multimodaler Therapiekonzepte soll berufspolitisches Glatteis verlassen werden und im folgenden die Frage nach der Zukunft der Operation im Rahmen der Onkologie näher eingegrenzt werden.

\section{Der Beitrag der Operation zur Krebsheilung}

Gemessen am Zielkriterium «Heilung» ist die Operation bis auf weiteres die erfolgreichste Modalität in der Onkologie. Trotz aller optimistischen Erwartungen an Radiotherapie, Chemotherapie, Immuntherapie und Gentherapie hat sich in der vergangenen Dekade an den Zahlen, die für die 80er Jahre hochgerechnet wurden, nicht grundsätzlich etwas geändert: Von allen Patienten, die tatsächlich von einem Krebsleiden geheilt werden, wurden in den 80er Jahren 62\% durch Chirurgie allein, 25\% durch Radiotherapie allein, 9\% durch eine multimodale Therapiekombination und $4 \%$ durch Chemotherapie allein geheilt [1].

Strittig und im Einzelfall heftig diskutiert ist die Frage, ob durch Verbesserung der chirurgischen Technik in den letzten Dekaden die onkologische Prognose tatsächlich verbessert wurde. Beispielhaft sei dies für den Stellenwert der erweiterten Lymphadenektomie beim Magenkarzinom ausgeführt. Ausgehend von den intensiven Bemühungen der asiatischen, insbesondere japanischen Chirurgen um eine Verbesserung der Prognose des Magenkarzinoms durch erweiterte Operationstechniken fand die erweiterte Lymphadenektomie in den 80er Jahren zunehmend Verbreitung auch in Europa. Dies führte

\begin{tabular}{ll}
\hline KARGER & @ 2000 S. Karger GmbH, Freiburg \\
Fax +49 7614520714 & Accessible online at: \\
$\begin{array}{l}\text { E-mail Information@Karger.de } \\
\text { www.karger.com }\end{array}$ & www.karger.com/journals/onk
\end{tabular}

Prof. Dr. med. Stefan Post

Universitätsklinikum Mannheim der Universität Heidelberg

Universitätsklinikum Mannheim

Tel. +49 621 383-22 25, Fax -3809

E-mail stefan.post@chir.ma.uni-heidelberg.de 
bis zu dem Punkt, daß die erweiterte Lymphadenektomie bereits zum nationalen Standard erklärt wurde [2]. Sowohl japanische als auch europäische Autoritäten begründeten aber die postulierten Prognoseverbesserungen durch erweiterte Radikalität der Lymphadenektomie mit «weichen» Daten aus großen retrospektiven Analysen, die unter dem Aspekt der Stadienmigration sehr kritisch zu bewerten sind [3]. Das Evidenzdefizit $\mathrm{zu}$ dieser Frage konnte mittlerweile verringert werden durch zwei größere randomisierte europäische Multicenter-Studien, die beide zu dem Ergebnis kamen, daß sich durch die generalisierte D2-Lymphadenektomie die Heilungsrate beim Magenkarzinom nicht verbessern lässt [4, 5]. Auch wenn beide Studien durch relativ hohe Komplikations- und Mortalitätsraten nach erweiterten Resektionen geschwächt werden [6], besteht hiernach wenig Hoffnung, allein durch Modifikation der chirurgischen Technik die Heilungsraten beim Magenkarzinom entscheidend zu verbessern.

Für die meisten anderen Organtumoren sind Fragen der Operationstechnik im allgemeinen und der Radikalität im speziellen noch schlechter untersucht [7]. Im Gegensatz zur Situation bei vielen Chemotherapien steht die «Evidence-Based Medicine» in der Chirurgischen Onkologie noch auf schwachen Füßen. Bisher weist wenig darauf hin, daß die Zukunft dramatische Fortschritte in der Tumor-Heilungsrate durch Ausweitung der chirurgischen Radikalität bringen kann. Eine Extrapolation der Tendenzen der vergangenen Dekaden spricht viel eher für eine Rücknahme früherer Radikalitätsansprüche.

Unzweifelhaft ist für die meisten Tumoren, daß die Prognose abhängig vom Tumorstadium ist. Je früher das Tumorstadium, um so eher kann nicht nur die langfristige Heilung erreicht werden, sondern auch auf den Einsatz multimodaler Therapiemaßnahmen verzichtet werden: Bei frühen Tumorstadien ist die mechanische Tumorentfernung durch Operation oder minimal-invasive Abtragung die beste Gewähr auf Heilung. Sollte sich - was zu erwarten ist - in den nächsten Dekaden die Rate der in Frühstadien erkannten Karzinome durch verbesserte Techniken (genetische Beratung, DNA-Chips, ScreeningProgramme) erhöhen, wird dies den Anteil derjenigen Patienten erhöhen, die allein chirurgisch unimodal behandelt werden können.

Der entscheidende chirurgische Fortschritt der letzten 20 Jahre liegt für die großen Organtumoren nicht in einer Verbesserung der stadienkorrigierten Heilungsrate. Vielmehr ist eine dramatische Senkung des perioperativen Risikos, vor allem hinsichtlich perioperativer Letalität, zu verzeichnen. Alle großen abdominellen Organresektionen bis hin zur Duodeno-Pankreatektomie, Gastrektomie und großen Leberresektion lassen sich mit Operationsletalitäten weit unter 5\%, meist von 1-2\% durchführen, vorausgesetzt, Operationsfrequenz und Erfahrung im perioperativen Management des jeweiligen Zentrums sind ausreichend groß $[6,8]$. Damit sind auch große Operationen in den Risikobereich gekommen, wo sie selbst palliativ zu rechtfertigen sind. Wegen Verbesserungen in Operationstechnik, Anästhesie, Intensivmedizin und Komplikationsmanagement wird in den nächsten Jahren und Jahrzehnten zu erwarten sein, daß die Zahl der operierten und insbesondere resezierten Patienten zunehmen wird. Aufgabe für die Chirurgie in ihrem ureigenen Teilbereich wird die Behebung der obenge- nannten Evidenzdefizite hinsichtlich Radikalität und die Umsetzung des an Spitzeninstitutionen erreichten niedrigen Operationsrisikos im Rahmen flächendeckender Qualitätssicherungsmaßnahmen sein. Für besonders anspruchsvolle Operationen mag dies nur durch Konzentration in spezialisierten Zentren realisierbar sein.

\section{Chirurgie im Rahmen multimodaler Konzepte}

Es ist allgemeiner Konsens, daß die Heilungsrate für fortgeschrittenere Stadien einiger Organtumoren in den letzten Jahren durch multimodale, insbesondere adjuvante Therapie verbessert werden konnte. Paradebeispiel ist das kolorektale Karzinom. Hier geht nach etlichen prospektiv randomisierten Multicenter-Studien der zur Zeit akzeptierte Standard dahin, daß das nodal-positive Kolonkarzinom (Stadium III) einer postoperativen adjuvanten 5-FU-basierten Chemotherapie zuzuführen ist. Gerade diese Empfehlung wird im Licht der schon oben diskutierten Evidenzdefizite der onkologischen Operationstechnik derzeit neu diskutiert: Alle großen randomisierten Studien zu dieser Fragestellung hatten keine ausreichende Standardisierung von chirurgischer Therapie (Lymphadenektomie etc.) und pathologischer Aufarbeitung der Präparate [9]. Ein - problematischer - Vergleich von «guten» Chirurgen mit der Operation allein und «schlechten» Chirurgen mit adjuvanter Therapie legt nahe, daß der 5-JahresÜberlebensgewinn durch adjuvante Chemotherapie von 12-16 Prozentpunkten nur in solchen Fällen zu erzielen ist, in denen der Chirurg nicht sauber gearbeitet hat. Auch national konnte selbst an engagierten Zentren belegt werden, daß eine hohe lokoregionäre Rezidivrate von um die $20 \%$ (wie in der klassischen Moertel-Studie [10]) mit einer Verschlechterung der 5-Jahres-Überlebensrate von 10-15\% einhergeht [11]. Deshalb wird diese Frage nun neu aufgeworfen in einer derzeit noch laufenden randomisierten deutschen MulticenterStudie. Ähnliches gilt für höhere Stadien des Rektumkarzinoms, wo sich (bisher nur in nichtrandomisierten Serien) durch optimierte chirurgische Technik (totale mesorektale Exzision) Rezidivraten erreichen lassen, die in klassischen Studien nur durch adjuvante Radio-/Chemotherapie zu erzielen waren.

Deshalb liegt es nahe und ist aus chirurgischer Sicht überfällig, daß Fragen der Notwendigkeit adjuvanter multimodaler Therapiemaßnahmen im Lichte verbesserter Operationstechniken neu aufzuwerfen sind. Die chirurgischen Onkologen lernen aus diesen Erfahrungen, daß sie sich in künftige multimodale Therapiestudien von Anfang an mehr als bisher einbringen müssen, insbesondere was die Standardisierung und Qualitätssicherung der chirurgischen und pathologischen Technik betrifft. Solange die Evidenzdefizite zur chirurgischen Technik nicht behoben sind, werden die akzeptierten Standards weiter durch - aus chirurgischer Sicht - schlechte Studien definiert. Ob die von kolorektalen Chirurgen erwartete Ablösung einiger adjuvanter Chemotherapien durch «bessere» Operation eintritt, bleibt abzuwarten. Ungeachtet der verschiedenen Tumorbiologie mag für manche solide Tumoren das Mammakarzinom als Paradigma gelten. Hier sind die Chirurgen im Laufe der letzten Dekaden keineswegs arbeitslos geworden; 
wohl aber hat sich der Stellenwert der Operation im multimodalen stadienadaptierten Therapiekonzept in hohem Maße relativiert. Die Eingriffe wurden immer weniger radikal.

Für die Zukunft ist bei soliden Tumoren hinsichtlich chemound auch speziell immuntherapeutischer Maßnahmen nicht zu erwarten, daß mit Verbesserung dieser Therapiemodalitäten die Chirurgie überflüssig werden könnte. Vielmehr spricht gerade für die Immuntherapie die experimentelle und klinische Evidenz der Abhängigkeit von der «Tumorlast» dafür, daß auch bei rapiden Fortschritten der Chirurg immer noch gefordert sein wird: weniger im Sinne supraradikaler Resektionen als vielmehr mit der Aufgabe, die Tumorlast auf unter $10^{9}$ Zellen ( $<1 \mathrm{~cm}$ Durchmesser) zu drücken [12].

\section{Ausblick}

Trotz immer wiederkehrender futurologischer Spekulation, daß das «Krebsproblem» in einigen Jahren durch neue Chemotherapeutika, durch Gentherapie, durch Immuntherapie oder ähnliches gelöst sein könnte, besteht derzeit keine erkennbare «Gefahr», daß onkologische Chirurgen arbeitslos werden könnten (oder sich mangels Einsatzmöglichkeit des Skalpells auf Plasmide stürzen müßten [13]). Folgende Gründe lassen sich dafür anführen:

- Demographie: Die meisten Krebserkrankungen sind Krankheiten des höheren Alters. Mit dramatischen demographischen Verschiebungen hin auf einen größeren Anteil der älteren Generation, vor allem in den Industrienationen, ist mit einer populationsbezogen höheren Karzinom-Inzidenz $\mathrm{zu}$ rechnen. Hinzu kommt noch, daß sich zumindest in Europa derzeit nicht absehen läßt, daß der Konsum kanzerogener Genußmittel oder Nahrungsbestandteile dramatisch sinken würde.

- Primärprävention: Von allen in Frage stehenden medizinischen Entwicklungen ist diese sicherlich die wünschenswerteste und diejenige, die den Chirurgen am ehesten über- flüssig machen könnte. Auch die größten Optimisten glauben aber kaum, daß in den nächsten Jahrzehnten für die meisten Organtumoren eine Primärprävention die Neuinzidenz maligner Tumoren nennenswert senken könnte.

- Screening: Hierzu wurde bereits oben aufgeführt, daß verbessertes Screening den Anteil der allein operativ therapierten Tumoren sogar erhöhen wird und deshalb zu einer Steigerung der Nachfrage nach operativer Therapie führt.

- Sicherheit der Eingriffe: Auch hierzu wurde oben bereits angeführt, daß mit zunehmender Sicherheit auch größere Eingriffe immer mehr Patienten zugemutet werden können.

- Tumorlast: Die Chirurgie bleibt die schnellste und effektivste Reduktion der Tumorlast, eventuell vor anderen Therapiemaßnahmen.

- Staging: Trotz erheblicher Fortschritte in der Bildgebung wird es zumindest in den nächsten 10 Jahren noch so bleiben, daß das exakte histopathologische Staging, das die operative Entfernung eines Tumors erlaubt, durch nichts $\mathrm{zu}$ ersetzen ist. Da sich für viele Organtumoren die therapeutischen Konzepte immer weiter im Hinblick auf eine stadienadaptierte Therapie differenzieren, wird der Stellenwert des exakten (chirurgisch-pathologischen) Stagings um so größer werden.

Zum Abschluß sei ein Zitat eines Molekular-Biochemikers angeführt, das eindrücklich nahelegt, daß auch in den Visionen von Nichtchirurgen die mechanische Tumorentfernung unverändert bestehen bleibt:

'In 25 years time the combination of the "2005 global smokingban act" and widespread adoption of chemopreventive dietary supplements, combined with annual whole-body health scans based on new imaging methods and DNA-chip-based mutation screens of blood, urine, stool, and breast effusions, will have halved the incidence of malignant metastatic cancer in the population. Accurate imaging, precise limited-access robotic surgery, and pre and post surgical systemic therapies and early treatment will have reduced the incidence of recurrence even further.' [14]

\section{Literatur}

\footnotetext{
1 Hoekstra HJ, Wobbes T, van de Velde CJ: The future of surgical oncology. Eur J Surg 1993;159:589-592.

2 Siewert JR, Stein HJ, Böttcher K: Lymphadenektomie bei Tumoren des oberen Gastrointestinaltrakts. Chirurg 1996;67:877-888.

3 Fass J, Hungs M, Reinecke T, Schumpelick V: Der Einfluß der D2-Lymphadenektomie auf Staging und Prognose des Magenkarzinoms - Eine prospektive Studie. Langenbecks Arch Chir Suppl Kongressbd 1996;113:176-178.

4 Bonenkamp JJ, Hermans J, Sasako M, van de Velde CJ: Extended lymph-node dissection for gastric cancer. Dutch Gastric Cancer Group. N Engl J Med 1999;340:908-914.

5 Cuschieri A, Weeden S, Fielding J, Bancewicz J, Craven J, Joypaul V, Sydes M, Fayers P: Patient survival after D1 and D2 resections for gastric cancer: Long-term results of the MRC randomized surgical trial. Surgical Co-operative Group. Br J Cancer 1999; 79:1522-1530.

6 Post S, Samel S, Becker H: Zum Risiko der Gastrektomie. Viszeralchirurgie 1998;33:89-95.

7 Köhler L, Eypasch E, Holthausen U,Troidl H: Myths in the management of colorectal malignancy. $\mathrm{Br} \mathrm{J}$ Surg 1997;84:248-251.

8 Sosa JA, Bowman HM, Gordon TA, Bass EB, Yeo CJ, Lillemoe KD, Pitt HA, Tielsch JM, Cameron JL: Importance of hospital volume in the overal management of pancreatic cancer. Ann Surg 1998; 228: 429-438.

9 Wein A, Riedel C, Lehnert T, Wakker R, Reingruber B, Teichmann W, Kohnen R, Herfarth C, Hohenberger W, Hahn EG: Adjuvante Behandlung beim Kolonkarzinom. Resultate und Perspektiven. Dtsch Med Wochenschr 1998;123:674-680.

10 Moertel CG, Fleming TR, Macdonald JS, Haller DG, Laurie JA, Goodman PJ, Ungerleider JS, Emerson WA, Tormey DC, Glick JH, Veeder MH, Maillard JA: Levamisole and fluorouracil for adjuvant therapy of resected colon carcinoma. N Engl J Med 1990;322:352-358
}

11 Hermanek P Jr, Wiebelt H, Riedl S, Staimmer D, Hermanek P: Langzeitergebnisse der chirurgischen Therapie des Coloncarcinoms. Ergebnisse der Studiengruppe Kolorektales Karzinom (SGKRK). Chirurg 1994;65:287-297.

12 Berd D: Cancer vaccines: Reborn or just recycled? Semin Oncol 1998;25:605-610.

13 Hollingsworth SJ, Barker SGE: New technologies: Gene therapy: Into the future of surgery. Lancet 1999;353(suppl I):19-20.

14 Lane D: The promise of molecular oncology. Lancet 1998;351(suppl II):17-20. 